Annuaire du Collège de France 2017-2018

\title{
Histoire intellectuelle de la Chine
}

\section{Anne Cheng}

\section{OpenEdition}

Journals

Édition électronique

URL : https://journals.openedition.org/annuaire-cdf/15776

DOI : 10.4000/annuaire-cdf.15776

ISBN : 978-2-7226-0572-5

ISSN : 2109-9227

Éditeur

Collège de France

Édition imprimée

Date de publication : 30 décembre 2020

Pagination : 335-350

ISBN : 978-2-7226-0516-9

ISSN : 0069-5580

Référence électronique

Anne Cheng, " Histoire intellectuelle de la Chine », L'annuaire du Collège de France [En ligne], 118| 2020 mis en ligne le 01 avril 2021, consulté le 22 août 2022. URL : http://journals.openedition.org/annuairecdf/15776 ; DOI : https://doi.org/10.4000/annuaire-cdf.15776 


\title{
HISTOIRE INTELLECTUELLE DE LA CHINE
}

\author{
Anne CHENG \\ Professeure au Collège de France
}

Mots-clés : civilisation, universalité, relativisme culturel, Xiongnu, Inde, Chine, Japon, Tianzhu, Bouddha, Laozi, Mouzi, Faxian

La série de cours « Universalité, mondialité, cosmopolitisme (Chine, Japon, Inde) (suite) 》 est disponible, en audio et vidéo, sur le site internet du Collège de France (https://www. college-de-france.fr/site/anne-cheng/course-2017-2018.htm), ainsi que le colloque «Autour du Traité des rites ( $L i j i)$ : de la canonisation du rituel à une société ritualisée » (https://www.college-de-france.fr/site/anne-cheng/symposium-2017-2018.htm).

\section{ENSEIGNEMENT}

COURS - UNIVERSALITÉ, MONDIALITÉ, COSMOPOLITISME (CHINE, JAPON, INDE) (SUITE)

L'année 2017-2018 a été consacrée au troisième volet d'un cycle de cours qui prend le parti de décentrer la civilisation chinoise, entreprise à la portée actuelle s'il en fut, à l'heure où la Chine se présente - et est perçue par le reste du monde comme étant en pleine montée en puissance et en position de faire prévaloir une prétention à l'universalité qu'elle aurait exercée sur sa périphérie pendant des siècles. Nous avons commencé par examiner ce qui a fait, à mon sens, le fondement d'une telle universalité, à savoir ce que j'ai appelé le «continuisme anthropo-cosmique de la culture ritualiste ». Nous nous sommes ensuite efforcés de montrer que ce continuisme est ancré dans une conception fortement centrée et hiérarchisée de la civilisation chinoise, conception qui se traduit par une représentation spatiale qui privilégie la figure géométrique hyper-stable du carré et le schéma du quadrillage. La civilisation chinoise se projette donc comme une centralité très forte qui irradie son influence par ondes concentriques sans qu'il y ait de limites extérieures. Nous avons tenté d'en dégager les fondements symboliques, illustrés dans des traces 
archéologiques, des sources textuelles et des formules devenues canoniques, dont on note une cristallisation à l'époque Han, dynastie qui a duré quatre siècles entre le $\mathrm{II}^{\mathrm{e}}$ siècle avant notre ère et le $\mathrm{II}^{\mathrm{e}}$ siècle de notre ère, et qui a consolidé l'unification territoriale et la centralisation réalisées par Qin. La représentation symbolique de la centralité Han est formulée dans diverses sources textuelles, notamment des textes canoniques ritualistes, à commencer par le Traité des rites (Liji 禮記). Rappelons le chapitre 《Mingtang wei 明堂位》(Les positions dans le Palais des lumières) qui donne une représentation spatialisée de la disposition hiérarchique de l'espace politique avec le Fils du Ciel au centre, entouré du carré des princes feudataires, en-dehors duquel sont disposés aux quatre orients les quatre peuplades «non civilisées » (Di au nord, Yi à l'est, Man au sud, Rong à l'ouest). Le chapitre «Wang zhi 王制》(Les institutions royales) dépeint leur situation marginale, en-dehors du pré carré des «pays centraux»(zhongguo 中國), ainsi que leurs mœurs qui les rapportent au non-humain et à l'animalité, telles que le fait de porter les cheveux dénoués et des tatouages sur le corps à la manière des démons, d'être vêtus de peaux de bêtes ou de plumes d'oiseaux, de ne pas se nourrir de céréales (faute de les cultiver comme les sédentaires) et de manger cru.

Yuri Pines, dans un article intitulé «Beasts or humans: Pre-imperial origins of Sino-Barbarian dichotomy ${ }^{1} »$, passe en revue les sources textuelles de la Chine préimpériale à la recherche de ce qu'il appelle le «beast simile », la métaphore de la bête sauvage, appliquée aux non-civilisés, en commençant par le Zhanguo ce 戰國 策 (Stratagèmes des Royaumes combattants), Wei ce 魏策 (Stratagèmes de Wei) :

秦與戎翟同俗, 有虎狼之心, 貪戻好利而無信, 不識禮義德行。苟有利焉, 不顧 親戚兄弟, 若禽獸耳。

[Les gens de] Qin [à l'ouest des pays centraux] ont les mêmes coutumes que les [barbares] Rong-Di, ils ont des cœurs de tigres et de loups, ils sont cupides et cruels, âpres au gain, sans foi ni loi, ils ne savent pas ce que sont les rites et le sens moral ni un comportement vertueux. Pour peu qu'ils voient quelque chose dans leur intérêt, ils font fi des liens de parenté, comme s'ils étaient des oiseaux et bêtes sauvages² ${ }^{2}$.

Toute la question est de savoir si ces Rong-Di sont considérés comme des nonhumains (c'est-à-dire ontologiquement autres, relevant d'une autre espèce plus proche de l'animal que de l'humain), ou bien si c'est simplement leur comportement qui est comparable à celui de bêtes sauvages (à noter la récurrence de ruo 若 = comme, comme si). Au fond, ce qui leur est reproché se borne à ce qu'ils « ne connaissent pas les rites » (bu shi liyi 不識禮義).

Un passage du Guoyu 國語 (Propos des principautés) est intéressant à cet égard et très révélateur de ce qu'il faut entendre par « les rites ». Il comporte des éléments de rhétorique qui oppose de manière édifiante les civilisés des «pays centraux » et les non-civilisés des marges. Il y est raconté comment un émissaire du puissant pays de Jin arriva à la cour royale des Zhou et se montra mécontent de la portion congrue de bouillon de viande qu'on lui servit au banquet sacrificiel. Le roi s'empressa de lui expliquer que dans les banquets royaux, c'est le respect des rites et non la quantité

1. Article paru dans le volume dirigé par Reuven AmITAI and Michal BIRAN, Mongols, Turks and Others: Eurasian Nomads and the Sedentary World, Leyde, Brill, 2005, p. 59-102.

2. Je souligne. 
des plats qui compte. Or, poursuivit le roi, ne pas comprendre ce principe, c'est se comporter comme les barbares Rong-Di :

\section{且唯戎狄則有體薦。夫戎狄, 冒沒輕俛, 貪而不讓。其血氣不治, 若禽獸焉。其 適來班貢, 不俟馨香嘉味, 故坐諸門外, 而使舌人體委與之。}

Il n'y a que les Rong-Di qui reçoivent la carcasse entière [de l'animal sacrifié au banquet]. Ces Rong-Di foncent tête baissée et dans le désordre, ils sont avides et ne cèdent pas la place (ils n'ont pas le sens rituel de l'effacement). Leur sang-énergie (xиeqi 血氣) n'est pas bien réglé, comme celui des bêtes sauvages (on retrouve ici la même expression que dans le Zhanguo ce). Lorsqu'ils viennent soumettre le tribut, ils ne sont même pas capables d'attendre le fumet odorant et le goût succulent des mets. Voilà pourquoi on les fait asseoir en-dehors des portes et on envoie les «hommes de langue » (qui peuvent communiquer avec les Rong-Di dans leur langue) traiter avec eux ${ }^{3}$.

L'absence de sens des rites chez les Rong-Di se voit à leur manque de retenue : comme les bêtes sauvages, ils se jettent sur la nourriture. On a ici la preuve a contrario de l'idée que les rites sont faits pour ralentir les gestes et le comportement, de manière à leur ôter leur caractère impulsif, voire violent. Ce qui rend les Rong-Di «comme des bêtes sauvages ${ }^{4}$ », c'est que leur sang-énergie n'est pas en bon ordre (ils ont le sang trop chaud), ce qui revient à dire que ce qui sépare l'humain de la bête est simplement une question de régulation du qi.

Comme à propos des Grecs, on a beaucoup dit et répété que la centralité Han a considéré « les autres » purement et simplement comme des « Barbares», en citant de façon presque routinière tous les textes où ces «autres » sont comparés à des 《oiseaux et bêtes sauvages» (ruo qin shou 若禽獸), ce que Yuri Pines appelle le «beast simile ». Mais comme le souligne Michael Nylan ${ }^{5}$, tout est dans le ruo 若, « comme » ou « comme si ». En effet, les textes ne disent pas que ces « autres » sont des bêtes sauvages, ils disent qu'ils se comportent comme des bêtes sauvages. Nulle part il n'est dit qu'ils ne sont pas des êtres humains, ou même qu'ils sont d'une espèce inférieure, l'accent est mis sur le fait qu'ils «ne connaissent pas les rites ». Autrement dit, à propos de ce que Pines appelle «Sino-Barbarian dichotomy » (dichotomie sino-barbare), faut-il parler de racisme ou de culturalisme? Le rapport entre les « centraux » et les « ex-centrés » ou, en termes chinois, la distinction Hua/ Yi, est-il d'ordre ontologique ou bien simplement culturel ? Si c'est ce dernier cas, comme semblent le dire la plupart des textes, du moins ceux d'inspiration confucéenne, on en déduit que les Yi ont la possibilité/capacité de devenir civilisés ou, du moins, de se laisser civiliser. Les maîtres confucéens sont, au demeurant, plutôt nuancés sur la distinction entre civilisés et non-civilisés. À en croire Mencius, la différence entre humains et animaux est extrêmement ténue, elle ne tient qu'à un fil qu'il incombe à l'humain de préserver, faute de quoi il a vite fait de retomber dans l'animalité, comme le résume le Mencius IV B 47 :

\section{孟子曰：人之所以異於禽於獸者幾希, 庶民去之, 君子存之。}

3. Gио уи 國語, Zhou yu 周語, zhong 中, § 20. Je souligne.

4. Je souligne.

5. Cf. «Talk about "Barbarians" in Antiquity » (compte rendu du livre d'Erich S. GRUEN, Rethinking the Other in Antiquity, Princeton, Princeton University Press, 2011), Philosophy East \& West, vol. 62, no 4, 2012, p. 580-601. 
Mencius dit : Ce qui différencie l'humain de la bête sauvage est d'une extrême ténuité : le commun des hommes a tôt fait de le perdre de vue, seul l'homme de bien sait le préserver.

Un autre passage fameux du Mencius (VI A 8) développe l'idée intéressante que la différence entre l'humain et le sauvage tient à la manière de contrôler l'énergie vitale, cette fameuse notion de $q i$ 氣 apparue dans les sources textuelles des IV $\mathrm{III}^{\mathrm{e}}$ siècles avant notre ère. Gardons en mémoire le texte du Guoyu cité plus haut, selon lequel le problème avec les Rong-Di est qu'ils ont le «sang chaud", littéralement un «sang-énergie pas bien réglé » (xueqi bu zhi 血氣不治):

Maître Meng (Mencius) dit : «Les arbres du Mont du Bœuf étaient beaux par le passé. Mais étant à proximité de (la capitale) d'un grand pays, on les coupe à la hache. Comment pourraient-ils conserver leur beauté ? Du fait du repos qu'ils prennent durant le jour et la nuit, et de l'humidité que leur apportent pluie et rosée, il leur repousse bien quelques surgeons, mais alors arrivent les bœufs et les moutons pour les brouter. Voilà pourquoi [la montagne] se retrouve dans un tel dénuement. En la voyant ainsi dénudée, on croit qu'elle n'a jamais eu de bois, mais comment cela pourrait-il être sa nature de montagne?

Quant à ce qu'on trouve en l'homme, comment pourrait-il n'y avoir aucun esprit d'humanité et de juste (ren yi 仁義, termes clés du vocabulaire confucéen) ? Ce qui fait que l'homme abandonne son esprit foncièrement bon est ce que la hache fait aux arbres ; si elle en coupe jour après jour, comment pourraient-ils garder leur beauté ? Du fait du repos qu'un homme prend durant le jour et la nuit, et de l'énergie ( $q i$ 氣) que lui procure le petit matin, ses attirances et aversions sont peu ou prou celles d'un être humain ; mais ensuite, ce qu'il fait pendant la journée l'amène à perdre [le bénéfice acquis pendant le repos]. À force de déperdition, son énergie accumulée pendant la nuit ne suffit plus pour subsister. Et quand elle est tarie, notre homme n'est plus très loin d'une bête sauvage. En voyant en lui une bête sauvage, on croit qu'il n'a jamais eu de potentialité [pour la bonté], mais est-ce là sa disposition naturelle d'être humain ${ }^{6}$ ?

Alors que les Confucéens conçoivent les non-civilisés comme des êtres qui ne connaissent pas les rites, les critiques des Confucéens, qui s'en prennent en premier lieu au ritualisme confucéen, expliquent pour leur part la «sauvagerie » par un relativisme culturel aux résonances parfois très modernes. Pour les Moïstes, il s'agit d'abord d'une question de conditions de vie et de différences de coutumes.

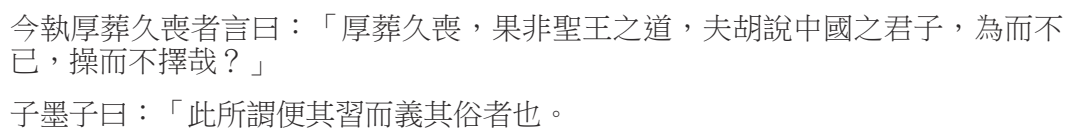

Ceux qui prônent des funérailles dispendieuses et un deuil prolongé ont ceci à dire :

«Ces funérailles dispendieuses et ce deuil prolongé, à supposer que ce n'était pas la Voie des Sages-Rois, comment expliquer que les hommes de bien des pays centraux (zhongguo 中國) les pratiquent sans discontinuer et les maintiennent sans discriminer? 》

Maître Mozi dit : «C'est ce qui s'appelle "trouver commode l'habitude" et "trouver juste la coutume" 7 ».

6. Je souligne.

7. Mozi 墨子, VI, 25, « Jie zang 節葬 (De l'économie dans les funérailles), xia 下», § 12. 
Suivent trois exemples pris chez des peuplades à l'est, au sud, et à l'ouest. À l'est de Yue, il est un pays où la coutume veut que le premier-né soit découpé en morceaux et mangé, afin de «favoriser les fils à naître ». Et quand le grand-père meurt, la grand-mère est emportée dans un endroit et abandonnée, au motif qu'on ne vit pas avec l'épouse d'un fantôme. Au sud de Chu, il est un pays où, quand un parent meurt, on laisse pourrir sa chair qui est ensuite jetée au rebut et on enterre ses os, en signe de piété filiale. À l'ouest de Qin, il est un pays où, quand un parent meurt, on ramasse du bois pour faire un grand feu sur lequel on brûle le corps, et lorsque la fumée s'élève, cela est appelé «l'ascension dans les nuées » et considéré comme le summum de la piété filiale. Ces trois exemples se terminent par une formule qui revient comme un leitmotiv :

此上以為政, 下以為俗, 為而不已, 操而不擇, 則此豈實仁義之道哉?

此所謂便其習而義其俗者也。

若以此若三國者觀之, 則亦猶薄矣。若以中國之君子觀之, 則亦猶厚矣。如彼則 大厚, 如此則大薄, 然則葬埋之有節矣。

Les gouvernants considèrent cela comme un mode de gouvernement, les gouvernés comme de la coutume. Ils le pratiquent sans discontinuer, et le maintiennent sans discriminer. Est-ce bien là la Voie de l'humain et du juste ? C'est seulement ce qui s'appelle «trouver commode l'habitude » et «trouver juste la coutume».

Si l'on considère les coutumes funéraires $d u$ point de vue de ces trois contrées (ex-centrées), elles sont par trop frustes. Mais si on les considère du point de vue des hommes de bien des pays centraux, elles sont par trop élaborées. Ces coutumes-là sont trop élaborées, ces coutumes-ci sont trop frustes, or il y a une juste mesure dans les règles d'inhumation ${ }^{8}$.

Un autre passage du Mozi est encore plus explicite dans sa critique relativiste :

魯陽文君語子墨子曰：「楚之南有啖人之國者橋, 其國之長子生, 則鮮而食之, 謂之宜弟。美，則以遺其君，君喜則賞其父。豈不惡俗哉？」

子墨子曰：「雖中國之俗，亦猶是也。殺其父而賞其子，何以異食其子而賞其父 者哉? 荷不用仁義, 何以非夷人食其子也?

Yang Wen, seigneur de Lu, dit à Maître Mo: «Au sud de Chu, il y a un pays de mangeurs d'hommes. Dans ce pays, lorsque naît le fils aîné, ils le mangent tout jeune, et appellent cela la façon juste d'honorer le cadet. Si la chair est succulente, ils en font offrande à leur prince, et si le prince est content, il récompense le père. N'est-ce pas là une coutume atroce? »

Réponse de Maître Mo: "Même si on prend les coutumes des pays centraux, on en trouve aussi de semblables [à celle des cannibales]. Mettre à mort le père et récompenser son fils, en quoi est-ce différent de manger le fils et récompenser le père ? Pour peu que l'on fasse abstraction du sens de l'humain et du juste, en quoi peut-on condamner les "sauvages" Yi au motif qu'ils mangent leur enfant ? ${ }^{9}$ »

Ainsi donc, à en croire Mozi, le cannibalisme de certaines populations « barbares » n'est pas pire que la condamnation à mort pratiquée dans les «pays centraux »

8. Je souligne.

9. Mozi, XIII, 49 «Lu wen 魯問 (Questions de Lu)». 
pourtant considérés comme centre de la civilisation, ce qui ne manque pas de nous rappeler le fameux chapitre «Des cannibales » des Essais de Montaigne (15531592) :

Or je trouve, pour revenir à mon propos, qu'il n'y a rien de barbare et de sauvage en cette nation [des Tupinambas du Brésil], à ce qu'on m'en a rapporté, sinon que chacun appelle barbarie ce qui n'est pas de son usage; comme de vrai, il semble que nous n'avons autre mire de la vérité et de la raison que l'exemple et idée des opinions et usances du pays où nous sommes. [...]

Je pense qu'il y a plus de barbarie à manger un homme vivant qu'à le manger mort, à déchirer par tourments et par géhennes un corps encore plein de sentiment, le faire rôtir par le menu, le faire mordre et meurtrir aux chiens et aux pourceaux (comme nous l'avons non seulement lu, mais vu de fraîche mémoire, non entre des ennemis anciens, mais entre des voisin et concitoyens, et, qui pis est, sous prétexte de piété et de religion), que de le rôtir et manger après qu'il est trépassé ${ }^{10}$.

Les réflexions de Montaigne sont inspirées par la concomitance de la découverte du Nouveau Monde et de ses « sauvages » (les Tupinambas du Brésil à qui l'on attribue des mœurs cannibales) et des guerres de religion en Europe et en France tout particulièrement (avec un paroxysme de violence dans la nuit de la Saint-Barthélémy, le 24 août 1572). Au moment même où les «Indiens » sont exhibés en spectacle pour le divertissement du roi de France et où font rage les guerres de religion en France, se tient en Espagne la fameuse controverse de Valladolid qui couvre presque une année, d'août 1550 à mai 1551, en présence d'une quinzaine de théologiens. Force est alors de décider si, oui et non, ces «Indiens» du Nouveau Monde sont des «fils de Dieu» comme les bons Chrétiens européens, et s'il est reconnu qu'ils le sont, il devient alors injustifiable de les réduire en esclavage ou à l'état de bêtes de somme.

L'affrontement entre les Han et les peuples des marges, à commencer par les nomades du Nord et de l'Ouest, notamment les Xiongnu 匈奴 (littéralement: esclaves), présente certains traits communs avec ce que Tzvetan Todorov a appelé la «question de l'autre », mais il est en réalité d'un autre ordre. Il est intéressant d'observer la manière dont la «métaphore de la bête sauvage» (ruo qinshou 若禽 獸) devient un poncif de la rhétorique guerrière qui justifie la politique de conquête et d'expansion menée par les Han, notamment par l'empereur Wu (le bien nommé « empereur guerrier» dont le règne de 141 jusqu'à sa mort en 87 avant notre ère fut l'un des plus longs et des plus marquants des Han antérieurs). Au chapitre consacré aux Xiongnu dans le Hanshu (Annales des Han antérieurs), nous voyons un morceau de bravoure aligner tous les poncifs et lieux communs concernant les « barbares » (à comparer avec le chapitre «Wang zhi » du Traité des rites mentionné plus haut) :

Les Yi-Di sont cupides et aiment le profit, ils laissent leurs cheveux dénoués et croisent leur tunique à gauche, ils ont des visages d'hommes mais des cœurs de bêtes sauvages (ren mian shou xin 人面獸心).

[...] Voilà pourquoi les anciens rois traitaient [les Yi-Di] comme des oiseaux et des bêtes sauvages (qinshou chu zhi 禽獸畜之), ne s'engageaient avec eux sur aucun traité ni aucun serment, et ne lançaient contre eux aucune expédition punitive. Si vous faites un traité avec eux, ils dilapident les présents et vous dupent; si vous les attaquez, ils

10. Essais, livre III, chap. 31, « Des cannibales ». Je souligne. 
épuisent vos troupes et appellent au brigandage. Leurs terres ne sont pas cultivables pour se nourrir, leurs gens ne sont pas traitables comme des sujets ${ }^{11}$.

Paradoxalement, cette tirade du Hanshu nous fait entrevoir une motivation à la fois politique et économique dans l'acharnement à tirer un trait de démarcation entre « pays centraux» et «barbares", entre civilisés et sauvages, entre sédentaires (cultivateurs) et nomades (cavaliers), mais aussi et surtout entre ceux de l'intérieur qui paient des impôts et ceux de l'extérieur qui sont forcés à payer le tribut en signe d'allégeance.

En outre, ce que ce magnifique exercice de rhétorique anti-barbare ne dit pas, c'est qu'en réalité, les Xiongnu, ces nomades vivant au nord et à l'ouest du monde Han, qui ont pu être plus tard assimilés aux Huns, étaient d'une puissance telle qu'ils étaient capables d'inquiéter et d'attaquer les Han, au point de devenir une menace permanente ${ }^{12}$. Face à ce puissant empire Xiongnu qui exige d'être traité d'égal à égal, il faut ruser. D'abord, en pratiquant une politique d'alliance par mariage (heqin 和親) ${ }^{13}$ engagée au tout début des Han sous l'empereur Gaozu, qui consiste à envoyer des princesses de la cour en échange de chevaux du Ferghana, et des cadeaux somptueux dont le prix dépasse de loin ce que peut rapporter en échange le « tribut ». Le but principal est d'obtenir la soumission de la part des royaumes de la périphérie sous la forme de visites d'émissaires à la cour des Han. Mais si le rapport de force n'est pas trop favorable, on se contentera d'obtenir a minima un signe symbolique d'allégeance sous la forme de l'octroi d'un titre nobiliaire ou d'un sceau. Attestent de ces difficultés les missions de longue durée du fameux Zhang Qian 張騫 (201113), envoyé vers l'ouest à deux reprises par l'empereur Wu et poussant toujours plus loin en Asie centrale, avec pour objectif de chercher des alliés contre les Xiongnu. Mais, ce faisant, le premier empire centralisé Han, qui a expérimenté toute la gamme des stratégies possibles vis-à-vis des «autres », en est arrivé à prendre conscience de l'existence possible au loin d'autres centres de civilisation.

Une jeune chercheuse américaine, Kaitlyn Ugoretz, dans un working paper intitulé "Distinguishing the "barbarian": Chinese exonyms and characterizations of the Other across Eurasia », livre des considérations intéressantes sur un glissement très net à l'époque Han dans le choix des noms (exonymes) utilisés pour désigner les populations non chinoises et dans les graphies de ces noms. L'assimilation avec l'animal transparaît dans la graphie de nombres de termes désignant les populations considérées comme non civilisées : par exemple, les Di 狄, dont le nom s'écrit avec le radical de l'animal, ou les Quanrong 犬戎, les «Rong-chiens»; ou bien les Qiang 姜 ou les Jie 羯, dont les noms comportent le radical du bouc 羊; ou encore les Man 蠻 dont le nom écrit avec le radical de l'insecte ou du rampant 虫 entre dans le composé courant dans la langue moderne yeman 野蠻 pour désigner tout ce qui est « sauvage » ou «barbare».

11. Hanshu 漢書, éd. Zhonghua shuju, p. 3833-3834.

12. Cf. H. VAN Ess, « The ethos of the envoy and his treatment by the enemy in Han history », Crossroads. Studies on the History of Exchange Relations in the East Asian World, vol. 5, 2012, p. 27-43.

13. Cf. chapitre 48 《He qin 和親》 (Traité de paix et d'alliance) du Yan tie lun 鹽鉄論, traduit par Jean LEVI sous le titre La Dispute sur le sel et le fer, Paris, Les Belles Lettres, coll. « Bibliothèque chinoise », 2010. 
En contraste total avec ces appellations discriminatoires figurent les noms de contrées lointaines et mythifiées par des adjectifs flatteurs à l'extrême, comme «grand» ( $d a$ 大) appliqué aux Da Yuezhi 大月氏 (parfois assimilés aux Kushans), à Da Yuan 大宛 (Ferghana), à Da Xia 大夏 (Bactriane) et à Da Qin 大秦 (Orient romain). Dans le même registre, la Sogdiane est appelée Kangju 康居 (Résidence prospère), la Parthie Anxi 安息 (Repos paisible), le pays des Kushan Guishuang 貴 霜 (Givre précieux). Quant au monde indien, Tianzhu 天竺, il est tout bonnement qualifié de «céleste» (tian 天). On constate donc que ces «autres au-delà des autres » ne sont pas traités à la même enseigne que les « autres » sur la périphérie immédiate de l'espace Han, et que cette distinction transparaît dans les exonymes utilisés pour désigner d'un côté les « sauvages à nos portes », et, dans une catégorie distincte, les autres qui sont loin, si loin qu'ils ne représentent pas une menace immédiate, mais bien plutôt un ailleurs exotique et fantasmé. On en voudra pour exemple cette phrase concernant les gens de Da Qin, extraite du chapitre consacré aux «Contrées occidentales » dans le Hou Han shu (Annales des Han postérieurs). Non seulement le lointain Orient romain est qualifié de «grand », mais il est ici très clairement assimilé à la Chine dont le nom vient précisément de Qin, la dynastie fondatrice du premier empire centralisé chinois ${ }^{14}$ :

\section{其人民皆長大平正，有類中國，故謂之大秦。}

Les gens y sont de haute taille et d'une parfaite équité. Il y a là quelque chose de comparable aux «pays centraux » (c'est-à-dire la Chine), voilà pourquoi ce pays est appelé Da Qin ${ }^{15}$.

Parmi ces «autres au-delà des autres » se détache la contrée dite «céleste », Tianzhu, autrement appelée Shendu/Yuandu 身毒 (par référence au Sind et à l'Indus), qui fait d'autant plus l'objet de fantasmes fabuleux qu'elle apparaît, de la même manière que l'Empire romain, comme un «autre » centre de civilisation, décrit comme peuplé de sédentaires qui cultivent la terre (par opposition aux « sauvages » nomades), qui disposent de riches ressources naturelles (métaux précieux, animaux rares, épices) et qui produisent des objets sophistiqués (textiles, parfums, sucre), qui montent des éléphants, qui n'aiment pas tuer ni aller à la guerre et qui « pratiquent la Voie du Bouddha (xiu Futu dao 脩浮圖道)» 16.

Le chapitre sur les contrées occidentales «Xiyu zhuan» du Hou Han shu se termine sur un épilogue (lun 論) :

至於佛道神化, 興自身毒, 而二漢方志莫有稱焉。張騫但著地多暑垒, 乘象而 戰, 班勇雖列其奉浮圖, 不殺伐, 而精文善法導達之功靡所傳述。余聞之後說 也，其國則殷乎中土 $[\ldots]$

14. Voir à ce propos Yü YING-SHIH, «Han Foreign Relations», in D. TwitchetT et M. LOEwE (dir.), The Cambridge History of China, vol. 1: The Ch'in and Han Empires, 221 $B C-A D$ 220, Cambridge, Cambridge University Press, 1986, p. 379.

15. Hou Han shu 後漢書 (Annales des Han postérieurs), «Xiyu zhuan 西域傳», § 25.

16. Cf. Hou Han shu, rédigé au ve siècle de notre ère, consacre un chapitre aux «contrées de l'ouest » (《Xiyu zhuan 西域傳 ») qui se fonde sur un document composé aux environs de l'an 125. La mention de Shendu/Yuandu 身毒 apparaît déjà dans le Shiji (Mémoires historiques) et le Hanshu (Annales des Han antérieurs). 
漢自楚英始盛齋戒之祀, 桓帝又修華蓋之飾。將微義未譯, 而但神明之邪? 詳其 清心釋累之訓, 空有兼遣之宗, 道書之流也。

Quant à la transformation divine opérée par la Voie du Bouddha, elle se développa à partir du Shendu/Yuandu, et pourtant dans aucun des traités sur les contrées [de l'ouest] dans les deux livres des Han (Shiji et Hanshu) il n'en est fait mention. Zhang Qian a seulement noté que cette terre est chaude et humide, et qu'on y monte des éléphants pour aller au combat. Ban Yong, bien qu'il ait exposé que les gens [de Shendu] adhèrent au Bouddha et s'abstiennent de tuer et de livrer bataille, n'a rien transmis ni relaté sur les mérites des beaux textes (sûtra) et de la bonne Loi (Dharma) à guider [les esprits] et leur faire atteindre [l'illumination]. Or, moi j'ai entendu dire à ceux qui en ont parlé par la suite que ce pays [de Shendu] est plus florissant que la Terre du milieu (la Chine).

[...] Sous les Han, depuis le règne de Ying de Chu, commencèrent à être en vogue les offrandes d'interdits liés au jeûne (en remplacement des sacrifices sanglants d'animaux), et l'empereur Huan (r. 147-167) adopta en outre l'ornement des dais fleuris [pour la statue de Bouddha]. Était-ce que les significations les plus subtiles n'étaient pas encore traduites et que seuls les esprits divins pouvaient les comprendre? Quant aux enseignements qui expliquent en détail comment purifier son cœur/esprit et se délivrer des liens [du désir], quant à l'origine du fait que la vacuité et la réalité sont à congédier l'un comme l'autre, tout cela dérive des écrits taoïstes ${ }^{17}$.

Cette dernière phrase témoigne d'un fait intéressant: à peine a-t-on reconnu l'existence d'une «autre» source de sagesse civilisatrice - en l'occurrence le Bouddha - que l'on s'empresse de lui trouver des origines chinoises. En témoigne également cet autre texte dû à Yu Huan 魚䅈 dans le Wei lüe 魏略 (Grandes lignes de l'histoire des Wei, composé entre 239-265), au chapitre sur les «Contrées occidentales »(《Xi yu 西域»):

臨兒國, 浮屠經云其國王生浮屠。浮屠, 太子也。父日屑頭邪, 母云莫邪。浮屠 身服色黃, 髮青如青絲, 乳青毛, 蛉赤如銅。始莫邪夢白象而孕, 及生, 從母左 脅出, 生而有結, 墮地能行七步。此國在天䇥城中。天䇥又有神人, 名沙律。

昔漢哀帝元壽元年，博士弟子景盧受大月氏王使伊存口受浮屠經日：復立者其人 也。浮屠所載臨蒲塞、桑門、伯聞、疏問、白疏間、比丘、晨門，皆弟子號也。 浮屠所載與中國老子經相出入, 蓋以爲老子西出關, 過西域之天䇥、教胡。

Concernant le pays de Linni (Lumbini, lieu de naissance du Bouddha dans l'actuel Népal), les livres canoniques de Futu (Bouddha) disent que son roi engendra Bouddha. Bouddha était le prince héritier. Son père s'appelait Xietouye (Suddhodana). Sa mère était appelée Moye (Mâyâ). Le Bouddha avait le corps recouvert d'une couleur dorée, ses cheveux étaient bleu-noir comme des fils de soie, sa poitrine couverte de poils bleunoir, sa langue (?) rouge comme du cuivre (références aux 32 lakșana, signes physiques distinctifs du Bouddha). À l'origine Mâyâ rêva d'un éléphant blanc et devint enceinte. À sa naissance, [le Bouddha] sortit du flanc gauche de sa mère. Dès sa naissance, il avait les cheveux noués en chignon (ushnisha). Ä peine eut-il touché terre qu'il fut capable de marcher sept pas. Ce pays se trouve au milieu des villes du Tianzhu. Au Tianzhu il y avait un autre homme divin, du nom de Shalü (Sâriputra, premier disciple du Bouddha).

Autrefois, dans la première année de l'ère Yuanshou du règne de l'empereur Ai des Han antérieurs (2 av. J.-C.), le docteur au vaste savoir Jing Lu reçut les instructions orales de Yicun, émissaire du roi des Da Yuezhi (Kushans), qui lui récita les sûtra bouddhiques

17. Je souligne. 
selon lesquels le «réapparu » était cet homme (c'est-à-dire le Bouddha) ${ }^{18}$. D'après les sources bouddhiques, linpusai (mis pour yipusai 伊蒲塞, upâsaka: disciples laïcs), sangmen (ou shamen 沙門, śramana: moines, ascètes), bowen et shuwen (śrāvaka : quelqu'un qui a entendu la voix du Bouddha), baishuwen (śrāvaka «blanc », c'est-à-dire pur ou âgé), biqiu (bhiksu: moine entré dans les ordres), chenmen (Dvārapāla: Gardien de la porte), sont autant d'appellations de disciples.

Ce qui est rapporté par les sources bouddhiques présente des analogies et des différences avec le livre canonique de Laozi. On pense en effet que Laozi serait parti vers l'ouest en sortant des passes, qu'il aurait traversé les contrées de l'ouest jusqu'au Tianzhu et qu'il aurait enseigné les Hu (désignation générique pour les peuples du Grand Ouest).

La dernière partie de la citation rappelle une légende qui brode sur la biographie non moins légendaire de Laozi dans le Shiji (Mémoires historiques de Sima Qian) selon laquelle Laozi serait parti vers l'ouest, aurait laissé au gardien de la dernière passe un écrit de 5000 mots sur dao de (la Voie et sa vertu ou puissance, donnant lieu au texte portant ce titre), et aurait ensuite disparu «sans que nul ne sache ce qu'il est devenu ». Ce qui laisse toute latitude pour imaginer que : soit Laozi est en réalité parti vers l'ouest pour s'initier à l'enseignement du Bouddha qu'il aurait ensuite rapporté en Chine - c'est la version des bouddhistes -, soit qu'il est parti vers l'ouest d'où il est revenu en Chine sous la forme du Bouddha (ou bien le Bouddha n'aurait été qu'un disciple de Laozi), ce qui signifierait en somme que le bouddhisme n'est en fait que le taoïsme revenu de l'Inde - c'est la version des taoïstes, en particulier des sectaristes religieux de mouvements comme celui des Turbans jaunes (huang jin 黄巾) à la toute fin des Han postérieurs.

Il devait inévitablement se produire entre bouddhisme et taoïsme un amalgame, assorti d'une tentation de «récupération » réciproque. L'idée que le Bouddha ne serait autre qu'une réincarnation de Laozi trouve son apothéose dans le Sûtra sur la conversion des barbares (Hua hu jing 化胡經). Ce sûtra composé de fait vers 300 de notre ère pour les besoins de la cause fut l'objet d'une fameuse querelle qui devait rebondir au $\mathrm{IV}^{\mathrm{e}}$, puis aux $\mathrm{VI}^{\mathrm{e}}-\mathrm{VII}^{\mathrm{e}}$ siècles ${ }^{19}$.

En l'an 520, devant l'empereur Xiaoming des Wei du nord 魏明帝 (r. 516-528) se tint entre bouddhistes et taoïstes un débat pour déterminer lequel, de Bouddha ou de Laozi, venait en premier, textes (faux si nécessaire) à l'appui, la question de départ étant : «Le Bouddha et Laozi ont-ils vécu à la même époque ? » Fo yu Laozi tongshi yi bu 佛與老子同時以不. C'est ainsi que le faux Qingjingfa xing jing 清净法行經 (Sûtra destiné à promouvoir la Loi claire et pure) prétendait que « le Bouddha avait envoyé trois disciples en Chine pour transmettre ses enseignements et convertir le peuple. Le Bodhisattva Rutong 儒童 fut appelé Kong Qiu (Confucius) par les Chinois; le Bodhisattva Guangjing 光净 fut appelé Yan Yuan (disciple de Confucius) ; et Mahâkâsyapa 摩訶迦葉 (disciple du Bouddha) fut appelé Laozi ».

Un autre texte qui témoigne de la complexité et de l'ambivalence du processus de réception du bouddhisme en contexte chinois est le Mouzi lihuo lun 牟子理惑論 (selon la traduction de Béatrice L'Haridon, Maître Meou, Dialogues pour dissiper la

18. La syntaxe de cette phrase est problématique. Selon une autre interprétation, c'est plutôt Jing Lu qui aurait été envoyé chez les Yuezhi.

19. Sur la controverse concernant la «conversion des barbares », voir Kristofer SCHIPPER, «Purity and strangers: Shifting boundaries in Medieval Taoism », T'oung Pao, vol. 80, 1994, p. 61-81. 
confusion), qui aurait été composé dans l'extrême sud de la Chine à la toute fin des Han (fin du II ${ }^{\mathrm{e}}$ siècle de notre ère) par un lettré chinois converti au bouddhisme et appelé Maître Mou (ou Meou) ${ }^{20}$.

傳曰 : 北辰之星, 在天之中, 在人之北 - 以此觀之, 漢地未必為天中也 ·

佛經所說, 上下周極含血之類物皆屬佛焉 ·是以吾復尊而學之 $\cdot$

Comme le dit le commentaire, l'étoile polaire est au centre du ciel et au nord du monde des hommes. On voit par là que la terre des Han ne se trouve pas forcément au centre du ciel. Il est dit dans les Sūtra du Bouddha que de haut en bas et jusqu'aux confins du monde, tout être vivant tient du Bouddha. C'est ainsi que je me suis mis à vénérer et à étudier [cet enseignement] ${ }^{21}$.

Béatrice L'Haridon commente ainsi ce passage: «L'universalité de l'enseignement bouddhique, sa vision d'un monde où tous les vivants partagent une nature commune, la nature de Bouddha, semblent ainsi avoir joué un rôle déterminant pour Meou-tseu [Mouzi]. De manière significative, cette idée est amenée par une nouvelle vision du cosmos, illustrée dans une sentence apparemment extraite d'un commentaire aux Classiques suggérant que le centre du ciel n'est pas le même que celui du monde des hommes, du moins celui qui est connu et reconnu dans le monde des Han. Le centre est déplacé et n'apparaît plus comme évident. L'enseignement bouddhique vient ajouter à ce décentrement un étirement du monde moral, en l'étendant à tous les êtres vivants. » Béatrice L'Haridon souligne à ce propos que le Mouzi reflète le point de vue très excentré d'un lettré vivant à l'extrême sud de la Chine d'alors (actuel Tonkin au nord du Vietnam), ce qui explique que cet ouvrage soit considéré comme fondateur dans le bouddhisme vietnamien.

Tout autre est la situation au nord. Toutes les dynasties non chinoises qui se succèdent au nord après la chute des Han encouragent puissamment le développement du bouddhisme qui, étant comme elles d'origine étrangère, leur procure un fondement spirituel et une légitimité politique hors des valeurs chinoises traditionnelles. En 399, au moment où les Qin postérieurs contrôlent le nord de la Chine, le moine bouddhiste Faxian 法顯 (env. 337-422) quitte la Chine pour l'Inde en quête de la Loi bouddhique, inaugurant ainsi une longue tradition de pèlerinages. À son retour en 414 , il entreprend de traduire les textes qu'il a rapportés et qui concernent principalement les règles de la vie monastique (Vinaya). Le récit de son périple se trouve dans le Foguo ji 佛國記 (Mémoire sur les pays bouddhiques), donné ici dans l'excellente traduction de JeanPierre Drège aux Belles Lettres (2013). En français, la toute première traduction en langue européenne, datée de 1836, est due à Jean-Pierre Abel-Rémusat (1788-1832), premier titulaire de la toute première chaire en Europe à être consacrée à la Chine, fondée en 1814 au Collège de France. Voici le début de ce récit :

20. Cf. Meou-Tseu (= Maître Meou), Dialogues pour dissiper la confusion, présentation et traduction par Béatrice L'HARIDON, Paris, Les Belles Lettres, coll. « Bibliothèque chinoise », 2017.

Les avis sont très partagés sur l'authenticité du texte. Pour Erik ZÜRCHER (The Buddhist Conquest of China:The Spread and Adaptation of Buddhism in Early Medieval China, Leyde, Brill, $3^{\mathrm{e}}$ éd., 2007, p. 13-15), c'est un faux datant au mieux du ve siècle. En revanche, pour Paul Pelliot («Meou-tseu ou Les doutes levés», T’oung Pao, vol. 19, 1920, p. 255-433), c'est bien un texte de la fin des Han (fin du II ${ }^{\mathrm{e}}$ siècle), avis partagé par Béatrice L'Haridon.

21. Mouzi, argument 14, traduction Béatrice L'HARIDON, op. cit., p. XXVI-XXVII. 


\section{法顯昔在長安, 慨律藏殘缺. 於是遂以弘始一年, 歲在己亥, 與慧景、道整、慧應、慧嵬 等, 同契至天䇥尋求戒律.}

Faxian résidait jadis à Chang' an (ancienne capitale des Han antérieurs, alors capitale des Qin postérieurs). Il déplorait les lacunes de la Corbeille de la discipline (Vinaya 22). Pour cette raison, en la $1^{\text {re }}$ année Hongshi, qui était un an cyclique yihai (399), il prit l'engagement, avec Huijing, Daozheng, Huiying, Huiwei et d'autres, de se rendre au Tianzhu pour y chercher des ouvrages sur les préceptes et la discipline.

Cette décision de Faxian d'entreprendre le long et périlleux voyage jusqu'en Inde, considérée comme la source vive de l'enseignement bouddhique, manifeste une volonté inédite de décentrement qui se trouve confirmée dans un autre passage devenu l'objet de maintes discussions :

度河便到烏萇國. 其烏萇國是正北天竺也. 盡作中天竺語, 中天竺所謂中國. 俗人衣 服、飲食, 亦與中國同. 佛法甚盛.

En traversant le fleuve, [Faxian] arriva au pays de Wuchang (Udyāna, au nord du Penjab actuel). L'Udyāna, c'est vraiment le Tianzhu du Nord. Tout le monde y parle la langue du Tianzhu central. Le Tianzhu central est ce que l'on appelle le Pays du milieu (Zhongguo 中國). Les vêtements, le boire et le manger des gens du commun sont aussi les mêmes que dans le Pays du milieu (Zhongguo). La Loi bouddhique y est très florissante ${ }^{23}$.

Ces désignations de «Tianzhu du Nord » (bei Tianzhu) et de «Tianzhu central » (zhong Tianzhu) font référence à la distinction faite dans les sources de l'Inde ancienne entre cinq régions : l'Inde du Nord, du Sud, de l'Est, de l'Ouest et du Centre (celle du Centre, appelée en sanscrit Madhyadeśa, littéralement le «pays du milieu », étant pour les sources bouddhistes indiennes le pays du Bouddha, c'est-àdire Magadha dans la plaine du Gange). Le problème est que lorsqu' on lit en chinois Zhongguo, on ne peut pas s'empêcher de penser qu'il s'agit de la Chine. Toute la question est donc de savoir ce que désigne Zhongguo dans les deux occurrences du passage cité ci-dessus. La première semble désigner clairement Madhyadeśa, la terre du Bouddha décrite à plusieurs reprises comme un royaume idéal ${ }^{24}$. Mais dans la seconde occurrence, serait-ce que Faxian ne pensait à comparer le pays du Bouddha avec la Chine ? Les avis sont partagés : Janine Nicol opte pour la compréhension de Zhongguo dans les deux occurrences comme se référant à Madhyadeśa ${ }^{25}$, se rangeant ainsi du côté de l'interprétation d'Abel-Rémusat, à laquelle s'oppose David Jonathan Felt, pour qui Zhongguo se réfère bien à la Chine dans l'esprit de Faxian ${ }^{26}$.

Comme l'a remarqué Abel-Rémusat, Faxian semble présupposer que «la terre centrale » (Zhongguo) ne saurait être que Madhyadeśa, ce qui trouve confirmation

22. Le canon bouddhique est traditionnellement constitué de trois corbeilles (sanscrit Tripitaka, chinois San zang 三藏) : Vinaya (discipline), Sütra (textes sacrés) et Abhidharma (textes de doctrine).

23. Foguo ji, § 19.

24. Voir par exemple Fo guo ji, § 40.

25. Cf. "Outsiders: Medieval Chinese buddhists and the "Borderland Complex". An exploration of the eight difficulties », The SOAS Journal of Postgraduate Research, vol. 6, 2014, p. 27-48.

26. Cf. «De-Centering the Middle Kingdom: The argument for Indian centrality within Chinese discourses from the $3^{\text {rd }}$ to the $7^{\text {th }}$ century », UC Santa Barbara: Ancient Borderlands Research Focus Group, 2010, Retrieved from https://escholarship.org/uc/item/695817kw. 
dans le fait qu'il se dit invariablement originaire de «terres frontalières (ou marginales) »(biandi 邊地). De fait, Faxian et ses compagnons ne parlent jamais de leur patrie en termes de Zhongguo, ils préfèrent se référer à des dynasties chinoises quand ils répondent qu'ils viennent de la «terre de Qin» (Qindi 秦地, cf. § 37), de la «terre de Jin» (Jindi 晋地, cf. § 120) ou de la «terre de Han» (Handi 漢地, cf. § 59). La « terre centrale» du Bouddha est tellement idéalisée et le désespoir de nos moines chinois d'être nés dans une « terre frontalière » est tellement irrémédiable que Daozheng, l'un des compagnons de route de Faxian, prend la décision radicale de ne pas rentrer en Chine et de rester en terre bouddhique :

道整既到中國, 見沙門法則, 眾僧威儀, 觸事可觀, 乃追歎秦土邊地, 眾僧戒律殘缺. 誓言 : “自今已去至得佛, 願不生邊地.”故遂停不歸. 法顯本心欲令戒律流通漢地, 於 是獨還.

Quand Daozheng parvint dans les royaumes du centre [de l'Inde, Zhongguo] et quand il vit les règles du Dharma des śramana et les manières dignes des moines, impressionné par ce qu'il pouvait observer, il se remémora et déplora les manques qui affectaient les préceptes et la discipline des moines en Chine et dans les territoires frontaliers (Qintu biandi). Il fit le serment : «Désormais jusqu'à ce que je devienne bouddha, je fais le vœu de ne pas renaître dans un pays frontalier (biandi). » Il resta donc et ne s'en retourna pas [en Chine]. Faxian, dont l'intention originelle était que les préceptes et la discipline se répandissent en terre Han (Handi), s'en retourna donc seul ${ }^{27}$.

La décision de Daozheng est tout à fait représentative de ce qu'Antonino Forte a appelé le «borderland complex » (complexe de la terre frontalière ${ }^{28}$ ) qui a continué à affecter des générations de moines chinois jusqu'à celle des très fameux Xuanzang 玄焋 (602-664) et Yijing 義淨 (635-713), qui firent eux aussi le voyage jusqu'en Inde plus de deux siècles plus tard. Après eux, le « borderland complex » devait être surmonté par la transformation de la Chine en une nouvelle «terre centrale» du bouddhisme, impliquant un transfert massif de sa terre d'origine vers de nouvelles « terres frontalières ». Toujours est-il qu'il semble important et urgent, dans le monde d'aujourd'hui, de se souvenir de ces temps sans précédent où la centralité chinoise s'est trouvée contestée par des Chinois eux-mêmes en une expérience unique d'auto-décentrement par référence à un autre centre de civilisation et d'universalité.

\section{SÉMINAIRE - LECTURE DU TRAITÉ DES RITES (SUITE)}

Le séminaire a été consacré, comme ceux des cinq années précédentes, à la lecture suivie d'un chapitre du Traité des rites (Liji 禮記). Celui de cette année a retenu le chapitre intitulé « Modèle ou norme de conduite» («Biaoji » 表記). Classé par Liu Xiang, Liu Xin puis Zheng Xuan parmi les « traités généraux («Tonglun » 通論), au même titre que le célèbre "Zhongyong», habituellement traduit par «le Juste milieu », et le «Ziyi » 緇衣 ( « Les robes noires »), il se présente comme une série de 49 courts articles que les commentateurs des Han et des Tang ont regroupé en huit parties. Chacune d'entre elles est introduite par la formule : «Le Maître dit (ou : a

27. Foguo ji, § 114 .

28. Cf. A. ForTe, «Hui-chih (fl. 676-703 A.D.), A Brahmin born in China », Estratto da Annali dell'Instituto Universitario Orientale, vol. 45, 1985, p. 106-134. 
dit) ceci » (Zi yan zhi 子言之), tandis que chaque article est précédé de l'expression : « Le Maître dit » (Zi yue 子曰).

Comme le «Ziyi », ce chapitre fait un usage massif des Poèmes (Shi 詩) et des Documents (Shu 書), les citant généralement en conclusion d'un propos du Maître comme gage d'autorité, de vérification et d'illustration. Il s'en distingue par des citations plus nombreuses des Mutations ( $\mathrm{Yi}$ 易). Sans que l'on puisse être absolument formel, et en l'absence jusqu'à présent de découverte archéologique ayant mis au jour ce texte, il est cependant très plausible de dater les matériaux de ce chapitre (sinon leur arrangement), comme le Ziyi découvert à Guodian, du début du III ${ }^{\mathrm{e}}$ siècle av. J.-C.

Plusieurs ensembles peuvent être dégagés: le premier évoque de manière discontinue des questions rituelles et insiste sur la prudence et la gravité dans la conduite ; le deuxième reprend à nouveaux frais la notion d'humanité (ren 仁) avant de la coupler avec celle d'équité ( $y i$ 義) ; un troisième ensemble présente les mérites comparés des trois dynasties des Xia, des Shang-Yin et des Zhou dans de longs articles assez proches des formules recueillies sur ce sujet dans les Entretiens, se montrant toutefois beaucoup plus critiques sur la décadence des Yin; les articles suivants s'attachent à décrire le « service du prince » en dégageant les motivations et la marge de manœuvre du ministre ; un cinquième ensemble reprend des éléments épars de la première partie sur la prudence ou le rapport entre les paroles et les actes ; enfin, le dernier examine les liens entre les sacrifices et la divination. Il ressort de cette composition que le chapitre a été peu « réarrangé » par les éditeurs des Han.

La lecture s'est ensuite attachée à plusieurs points. Le titre « Biaoji », tout d'abord, que l'on peut traduire par «norme [de conduite]» nous a semblé pouvoir être interprété par contraste avec le sens de gnomon qu'il prend dans les écrits moïstes et où il désigne le critère de validité d'un discours. Dans ce chapitre, la norme est la conduite de l'homme de bien.

Le texte présente aussi une réflexion particulièrement riche sur la vertu d'humanité, assez différente de celle que développent les Entretiens. L'accent est davantage mis sur la dimension utilitariste de cette vertu. Dans la perspective qui est sans doute celle de Zisi puis de Mencius, elle est couplée non avec le sens des rites, mais avec l'équité.

Le séminaire a accueilli deux interventions. Jean Levi est venu présenter, peu de temps avant la parution d'une traduction conjointe des Entretiens et du Laozi dans la « Bibliothèque chinoise » des Belles Lettres, ses arguments contre le « négationnisme » de la sinologie américaine qui, depuis plus d'une trentaine d'années, fait des Entretiens un texte reconstruit, composé et pour partie écrit sous la dynastie des Han. Marc Kalinowski, directeur d'études émérite à l'École pratique des hautes études, a présenté la dernière partie du chapitre «Biaoji », consacrée à la divination.

\section{COLLOQUE INTERNATIONAL - AUTOUR DU TRAITÉ DES RITES (LIJI): DE LA CANONISATION DU RITUEL À UNE SOCIÉTÉ RITUALISÉE / \\ ALL ABOUT THE RITES: FROM CANONIZED RITUAL TO RITUALIZED SOCIETY}

Ce colloque, organisé par la chaire conjointement avec le Pr Stéphane Feuillas de l'université Paris-Diderot et avec le soutien de la fondation Hugot du Collège de France, s'est tenu les 21 et 22 juin 2018. Il a rassemblé des spécialistes venus 
d'Europe, des États-Unis, de Chine, de Taiwan, d'Australie, ainsi que des collègues du Collège de France et d'autres institutions françaises. Il s'agissait d'interroger à nouveaux frais la manière dont la Chine ancienne s'est progressivement constituée comme une société ritualiste, c'est-à-dire comme un monde dans lequel les interactions sociales en temps de paix devaient être principalement abordées à partir de la catégorie du rite. Si cette catégorie reste difficilement définissable de manière univoque, il est apparu que l'une des manières de l'aborder et d'en montrer l'étendue et les ressources était de le faire à partir de la canonisation, sous la dynastie des Han (206 av. J.-C.-220 ap. J.-C.), d'un compendium de textes qui ont pour but de régler les conduites, le rapport entre les vivants et les morts autant que les rapports de pouvoir ou le calendrier des activités humaines. Le premier objectif du colloque était donc d'examiner comment s'articulent ces textes entre eux dans l'établissement d'un corpus canonique et, plus précisément, de s'interroger sur l'incorporation de textes très composites comme le Traité des rites, qui a fait l'objet d'un séminaire de la chaire depuis cinq ans. Il s'agissait enfin de montrer par contraste avec d'autres traditions et approches sociologiques comment la canonisation du rituel en Chine a modelé le sens du rite et les formes du questionnement social et politique en Chine ancienne, et comment le rite continue de servir de fondement à certaines réflexions modernes sur l'organisation et la gestion des hommes.

\section{COURS À L'EXTÉRIEUR}

Janvier-février 2018 : deux cours et séminaires à Delhi University sur : «French orientalism between indology and sinology» et «The Chinese reception of the European concept of liberty $\gg$.

Avril 2018 : un cours et un séminaire à University of Chicago sur : «Chinese as a translation language and culture ».

Mai 2018: un cours et un séminaire à l'Académie autrichienne des sciences (Vienne) sur : « The place of philosophy in the history of Asian Studies. Reflections on China and India ».

\section{CONFÉRENCIERS INVITÉS}

Pr Watanabe Hiroshi (université de Tokyo), deux conférences données en français les 4 et 8 juin 2018 : «Structure du genre et sexualité dans le Japon de Tokugawa (1600-1867)» et «Tocqueville et les trois révolutions: France (1789- ), Japon (1867- ), Chine (1911- )».

Pr Chu Xiaoquan (université Fudan, Shanghai), une conférence donnée en français le 7 juin 2018 : « Mai 68 vu de Chine».

\section{RECHERCHE}

\section{Sanchit Kumar, ATER}

Sanchit Kumar a continué à m'assister dans la préparation de mon cours au Collège de France (recherche de documentation et de collaborations possibles, synthèse de publications, vérification de données bibliographiques, etc.), ainsi que dans l'organisation scientifique et pratique du colloque international sur les rites 
proposé par la chaire et tenu principalement en anglais au Collège les 21 et 22 juin 2018. Il a, par ailleurs, poursuivi son travail de thèse, tant pour les enquêtes de terrain que pour la rédaction de certains chapitres.

\section{Hengying Rong, ATER, boursière Anna Caroppo}

Dans le cadre de son contrat d'ATER au Collège de France abondé par la bourse Anna-Caroppo, Hengying Rong a aidé à l'accueil des participants (notamment de Chine continentale et de Taiwan) au colloque international sur le Traité des rites organisé en juin 2018. Elle a également contribué à la relecture de la traduction en chinois de mon Histoire de la pensée chinoise parue en mars 2018. Elle a, en outre, animé divers séminaires à l'ENS et à l'EHESS, tout en travaillant à l'achèvement de sa thèse prévu dans un an.

\section{PuBLICATIONS}

\section{LIVRES, ARTICLES ET CHAPITRES D'OUVRAGES}

Cheng A., « Comment hériter dans une Chine amnésique? », in J. BiRnBaum (dir.), Hériter, et et après ?, Gallimard, coll. « Folio Essais », 2017, p. 153-170.

Cheng A., «La place des droits de l'homme dans la pensée chinoise », Revue des deux mondes, février-mars 2018, p. 92-100.

CHEng A., "Central India is what is called the Middle Kingdom », in Anjana SHARMA (dir.), Records, Recoveries, Remnants and Inter-Asian Interconnections. Decoding Cultural Heritage, Singapour, ISEAS/Yusof Ishak Institute, 2018, p. 141-159.

CHENG A., «De la "Chine-monde" à la Chine du nouvel ordre mondial », Revue Défense nationale, vol. 811 (L'Empire du Milieu au cour du monde. Stratégie d'influence et affirmation de la puissance chinoise), 2018, p. 70-78.

Cheng A., « La Chine est-elle capable de penser ? », L'Archicube, vol. 24, 2018, p. 125-129.

CHENG A., « Is the dialogue of cultures a contemporary myth? », Culture and Dialogue, vol. 6, 2018, p. 51-60.

\section{TRADUCTIONS}

Participation à la traduction en chinois de mon Histoire de la pensée chinoise (traduction de Dong Yi et RoNG Hengying), Zhongguo sixiang shi 中國思想史, Presses universitaires du Henan (Henan daxue chubanshe 河南大學出版社), 2018.

\section{ÉdITION (DIRECTION DE COLLECTION)}

Du Fu, La Guerre civile (755-759). Euvre poétique II, présentation et traduction de N. ChAPUIS, Paris, Les Belles Lettres, 2018.

LaO-Tseu, Confucius, Les Deux arbres de la Voie : Le Livre de Lao-Tseu / Les Entretiens de Confucius, 2 vol., présentation et traduction de J. Levi, Paris, Les Belles Lettres, 2018. 